\title{
Optoelectrical Operation Stability of Broadband PureGaB Ge-on-Si Photodiodes with Anomalous Al-Mediated Sidewall Contacting
}

\author{
Max Krakers ${ }^{1} \cdot$ Tihomir Knežević $^{1,2}$ (D) Lis K. Nanver ${ }^{1,3}$
}

Received: 7 May 2021 / Accepted: 13 September 2021 / Published online: 5 October 2021

(c) The Author(s) 2021

\begin{abstract}
An anomalous aluminum-mediated material transport process was investigated in sets of Ge-on-Si photodiodes with broadband optoelectrical characteristics measured at wavelengths from $255 \mathrm{~nm}$ to $1550 \mathrm{~nm}$. The diodes had "PureGaB" anode regions fabricated by depositing a Ga wetting layer capped with an 11-nm-thick B-layer on $0.5 \mu \mathrm{m}$-thick Ge islands grown on $\mathrm{Si}$. The $\mathrm{Al}$ metallization was able to reach the $\mathrm{Ge}-\mathrm{Si}$ interface through $\sim 0.1-\mu \mathrm{m}$-wide holes inadvertently etched along the perimeter of the Ge-islands, and then traveled along the Ge-Si interface, displacing and recrystallizing $\mathrm{Ge}$ and $\mathrm{Si}$. The rest of the Ge surface was protected from the $\mathrm{Al}$ contact metallization by the B-layer. For diodes that had received the standard $400^{\circ} \mathrm{C}$ $\mathrm{Al}$ alloying step, the responsivity was near-theoretical at $406 \mathrm{~nm}$ and $670 \mathrm{~nm}$, but, at $1310 \mathrm{~nm}$ and $1550 \mathrm{~nm}$, the proximity of $\mathrm{Ge}-\mathrm{Si}$ interfacial defects caused significant attenuation. Extra annealing at $400^{\circ} \mathrm{C}$ or $500^{\circ} \mathrm{C}$ enhanced the formation of Si pits that were filled with modified Ge crystals alloyed with $\mathrm{Si}$ and $p$-doped with Al. All these diodes maintained low dark currents, below $50 \mu \mathrm{A} / \mathrm{cm}^{2}$ at $2 \mathrm{~V}$ reverse bias, but the responsivity was degraded, particularly for the long wavelengths. On the other hand, neither responsivity nor degradation of current-voltage $(I-V)$ characteristics was observed for prolonged exposure to normal operating temperatures up to $100^{\circ} \mathrm{C}$. Since the direct Al contacting of the Ge sidewalls does not degrade the dark current, for large diodes it could be a low-cost method of obtaining low contact resistance to an anode with $p$-type sidewall passivation and high fill-factor.
\end{abstract}

Keywords Aluminum-mediated material transport $\cdot$ broadband photodiodes $\cdot$ boron material barrier $\cdot$ gallium $\cdot$ Ge-on-Si diodes $\cdot$ near-infrared photodiodes $\cdot$ ultraviolet photodiodes

\section{Introduction}

We carried out an in-depth investigation of one of the critical processing steps in the fabrication of Ge-on-Si photodiodes with "PureGaB" anodes formed by capping Ge-islands with first a wetting layer of $\mathrm{Ga}$ and then a few nanometers of pure boron. Specifically, in the diodes studied here, and described earlier in, ${ }^{1,2}$ the contacting of the perimeter of the anode inadvertently resulted in the aluminum metallization being able to reach and react with the $\mathrm{Ge} / \mathrm{Si}$ interface.

Tihomir Knežević

tihomir.knezevic@fer.hr

1 MESA+ Institute of Nanotechnology, University of Twente, Enschede, The Netherlands

2 Faculty of Electrical Engineering and Computing, MINEL, University of Zagreb, Zagreb, Croatia

3 Department of Materials and Production, Aalborg University, Aalborg, Denmark
This in turn resulted in an anomalous Al-mediated material transport. Otherwise, the B-layer forms a material barrier between the $\mathrm{Al}$ and $\mathrm{Ge}$, preventing such interactions that occur at standard back-end CMOS processing temperatures around $400-500^{\circ} \mathrm{C}$. Also, for other metals, nanometer-thin B-layers have proven to be potent material barriers to semiconductors, ${ }^{3}$ and can replace more commonly used barrier materials like TiN. ${ }^{4}$

Despite the unwanted Al-Ge perimeter interaction in the PureGaB photodiodes studied here, the devices distinguished themselves by being responsive at far ultraviolet (FUV) wavelengths as low as $255 \mathrm{~nm}$, as well as having the short wavelength infrared (SWIR) sensitivity characteristic of Ge photodiodes, as already reported in. ${ }^{5}$ This broadband nature is due to the damage-free method with which the PureGaB anode is fabricated that gives a shallow, graded $p^{+}$-doping profile without role-off at the $\mathrm{Ge}$ surface. ${ }^{6,7}$ This enables low dark currents and photosensitivity close to the Ge surface. The absorption length of 
UV light is only about $10 \mathrm{~nm}$ for wavelengths around 200 $\mathrm{nm},{ }^{8}$ much lower than conventional photodiode junction depths. In CMOS, it is common to use specially designed $\mathrm{Si}$ photodiodes with ultrashallow junctions in this wavelength range. ${ }^{9}$ Compared to these Si photodiodes, the inherently much higher dark current of Ge photodiodes is a disadvantage. Nevertheless, a comparison of the optical constants and internal quantum yields of $\mathrm{Si}$ and $\mathrm{Ge}^{10-12}$ indicates that $\mathrm{Ge}$ photodiodes could even outperform $\mathrm{Si}$ diodes for the detection of FUV light if the photosensitive region of the $p n$-junction in $\mathrm{Ge}$ is brought close enough to the surface.

Alternative methods presented for forming shallow $p n$ junctions in Ge devices include ion implantation, ${ }^{13}$ monolayer doping, ${ }^{14}$ and spin-on-dopants activated by laser annealing. ${ }^{15,16}$ Defect-free $p n$-junctions are also grown epitaxially by chemical vapor deposition (CVD) in blanket depositions, but they require mesa etching for device isolation. ${ }^{17}$ Often these methods lead to high dark currents above $10^{-3} \mathrm{~A} / \mathrm{cm}^{2}$ due to material defects produced during the fabrication/isolation of the $p n$-junction. ${ }^{18}$ This is in contrast to PureGaB photodiodes that, in past reports, were demonstrated to have ideality factors less than 1.1 and dark current densities as low as $15 \mu \mathrm{A} / \mathrm{cm}^{2}$ at room temperature, ${ }^{1}$ and operation was achieved as avalanche photodiodes in both linear and Geiger modes. ${ }^{2,19}$ The dark current values are among the best reported in the literature. ${ }^{20-22}$ Moreover, PureGaB Ge-on-Si photodiodes were fabricated in $300 \times 1$ arrays with different pixel geometries showing high reproducibility and uniformity of the electrical and optical characteristics. ${ }^{2}$ Except for ${ }^{22}$ that presents a lateral Ge photodiode, the junction depth of other vertical photodiodes was large and responsivity values for wavelengths below $600 \mathrm{~nm}$ were not reported.

The broadband detection of wavelengths in the range 200-1600 nm coupled with CMOS compatibility holds a promise of increasing functionality in a variety of optoelectronic systems. For example, fields such as spectroscopy, ${ }^{23}$ military ${ }^{24}$ and security ${ }^{25}$ applications benefit from having a single broadband sensor to cover detection of photons from FUV to SWIR wavelengths. To profit from the potential boost in functionality, the broadband sensors need to be integrated with CMOS circuitry, preferably monolithically. This has, for example, led to work on the monolithic integration of graphene in CMOS as a phototransistor that forms the basic component of broadband image sensors. ${ }^{26}$ Nevertheless, seamless integration of materials such as graphene in CMOS is still technologically challenging. ${ }^{27}$ In contrast, Ge can be easily grown directly on silicon and is responsive to SWIR wavelengths up to $1600 \mathrm{~nm}$. In several Ge-on-Si technologies, the fabrication of high-performance photodetectors has been demonstrated, ${ }^{17,19,28,29}$, but they were not characterized at UV wavelengths. For PureGaB Ge-on-Si diodes, earlier focus was mainly on the CMOS compatibility and overall good performance.

In this paper, the anomalous Al-mediated material transport is investigated in relation to the degree to which it does or does not degrade the broadband optoelectronic PureGaB photodiode performance. The diode current-voltage $(I-V)$ characteristics and responsivity are determined using laser wavelengths from $406 \mathrm{~nm}$ to $1550 \mathrm{~nm}$. After the standard $400^{\circ} \mathrm{C} \mathrm{Al}$ alloying step, the devices were exposed to a series of extra anneals in the temperature range $100^{\circ} \mathrm{C}$ to $500^{\circ} \mathrm{C}$. To our knowledge, this is the first report describing this specific type of material transport system. Other systems have been studied with the purpose of achieving Al-induced crystallization of Ge or SiGe, ${ }^{30-34}$ mainly for the purpose of fabricating low-cost SWIR photodiodes. This work contributes to an understanding of the situations in which Al-mediated recrystallization of $\mathrm{Ge} / \mathrm{Si}$ material systems can be achieved.

The experimental procedures for device fabrication and optoelectrical measurement are described in "Experimental Methods" along with basic simulation parameters used to estimate the achievable responsivity. In "Results", the results of material analysis and optoelectrical measurements are given, and the overall results are discussed in the "Discussion", leading to the conclusions presented in the "Conclusions".

\section{Experimental Methods}

\section{Device Fabrication}

In Fig. 1, a schematic process flow for the fabrication of the PureGaB Ge-on-Si diodes is shown. They were integrated 2-5 ohm-cm $n$-type (100) 100-mm Si wafers covered with a layer-stack of 30-nm-thick thermal and $1-\mu \mathrm{m}$-thick lowpressure $\mathrm{CVD} \mathrm{SiO}_{2}$, and patterned with windows etched to the Si surface (Fig. 1a). All the depositions were performed in an ASM Epsilon 2000 CVD reactor equipped with germane $\left(\mathrm{GeH}_{4}\right)$, arsine $\left(\mathrm{AsH}_{3}\right)$, trimethylgallium (TMGa) and diborane $\left(\mathrm{B}_{2} \mathrm{H}_{6}\right)$ carrier gases. After dip-etching in $0.5 \%$ diluted hydrofluoric acid and Marangoni cleaning, ${ }^{35}$ the Ge was deposited in the windows to create islands with a thickness less than that of the surrounding oxide. These $\mathrm{Ge}$ islands were in situ $n$-doped with arsenic to a value of about $10^{16} \mathrm{~cm}^{-3}$ and, after growth, annealed at $750^{\circ} \mathrm{C}$ for $1 \mathrm{~h}$. The defect density of the crystalline Ge that was deposited with this approach was shown to be in the order of $10^{7} \mathrm{~cm}^{-2}, 36$ and the resulting Ge photodiode islands within our $300 \times 1$ diode arrays displayed a high reproducibility with respect to the resulting optoelectronic characteristics. ${ }^{1,2}$

The Ge-islands were capped in situ with a wetting-layer of $\mathrm{Ga}$ at $400^{\circ} \mathrm{C}$ and then a B-layer at $700^{\circ} \mathrm{C}$ that functioned as a barrier to prevent $\mathrm{Ga}$ desorption and interactions 
(a)

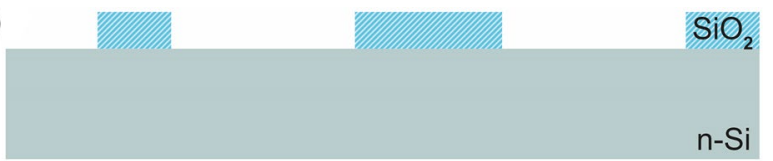

(b)

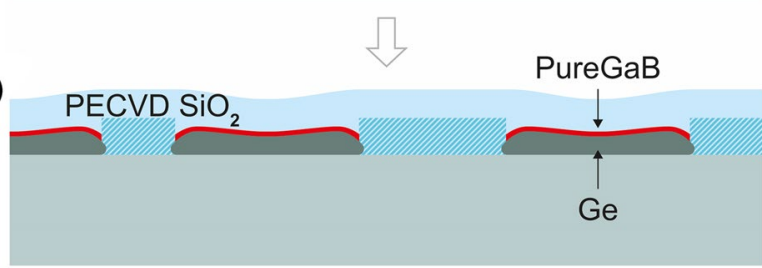

(c)

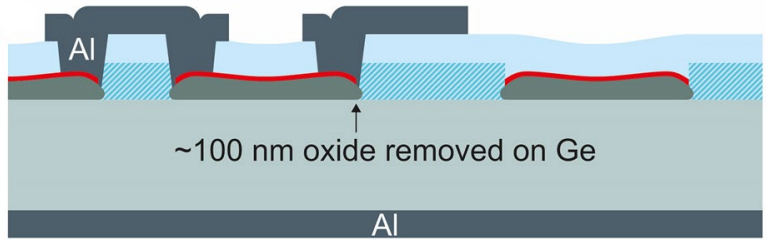

Fig. 1. Schematic process flow for the fabrication of the PureGaB Ge-on-Si photodiodes: (a) oxide window opening for selective epitaxial $\mathrm{Ge} / \mathrm{Ga} / \mathrm{B}$ growth; (b) plasma-enhanced chemical vapor deposition (PECVD) of $\mathrm{SiO}_{2}$; and (c) contact window etching, and $\mathrm{Al} / \mathrm{Si}(1 \%)$ sputtering and patterning.

between the $\mathrm{Ge}$ and $\mathrm{Al}$ metallization. This Ga-B layer stack was $11 \mathrm{~nm}$ thick, as extracted from energy dispersive $\mathrm{x}$-ray spectroscopy (EDS) in, ${ }^{1}$ and formed the anode of the photodiodes. The wafers were then covered with a 800 -nm-thick plasma-enhanced CVD $\mathrm{SiO}_{2}$ layer (Fig. 1b), which was patterned with $1-\mu \mathrm{m}$-wide windows along the perimeter of the Ge-photodiode islands to contact the anode. These windows were opened by plasma etching with soft landing on the B-layer. Next, $800-\mathrm{nm} \mathrm{Al} / \mathrm{Si}(1 \%)$ was sputtered and removed over the photosensitive junctions by means of selective plasma etching of the $\mathrm{Al}$ layer to the oxide covering the Ge-islands (Fig. 1c).

As a last step, the metal was alloyed at $400^{\circ} \mathrm{C}$ in forming gas to improve the $\mathrm{Al}$ adhesion and also $\mathrm{H}$-passivate the $\mathrm{Si} / \mathrm{SiO}_{2}$ interface. To study the effects of this temperature treatment in more detail, fully processed devices were optoelectronically characterized before and after extra heat treatments at $100^{\circ} \mathrm{C}$ and $500^{\circ} \mathrm{C}$ performed in a bell-jar setup with a vacuum less than $10^{-3}$ mbar.

\section{Device Design}

Measurements were performed on Ge photodiodes with a variety of geometries, some of which are listed in Table I. The sizes of individual square-shaped Ge-islands ranged from $5 \times 5 \mu^{2}$ to $26 \times 26 \mu \mathrm{m}^{2}$, and $50 \times 11 \mu^{2}$ devices were also available. To prevent loading effects during the depositions from giving a large size dependence of the Geisland thickness and doping, 5 - $\mu \mathrm{m}$-wide open $\mathrm{Si}$ windows were patterned to form a moat around the device islands, as shown in Fig. 1. The selectivity of the $700^{\circ} \mathrm{C}$ depositions was good and no precipitates of $\mathrm{Ge}$ are found on the oxide surface after $\mathrm{Al}$ etching, neither by optical/material examination nor electrical measurement.

Two different process runs were studied that differed in the length of the $400^{\circ} \mathrm{C}$ alloy step. As indicated in Table I, the SPD diodes had a short 30-min alloy while the LPD diodes received a longer 1-h alloy. Some diodes were composed of groups of Ge-islands separated by a $1-\mu \mathrm{m}$-wide oxide region. Therefore, the ratios between Ge perimeter/ area and the total metal coverage varied significantly, as specified in Table I.

\section{Measurement Techniques}

The main aim of the optoelectrical measurements was to couple the electrical low-voltage $I-V$ diode characteristics to the corresponding optical responsivity. These results were supplemented with images taken by optical microscopy and atomic-force microscopy (AFM). In addition, high-resolution transmission electron microscopy (HRTEM) images of the SPD devices, some previously discussed in Ref. 1, were also available.

Optoelectronic measurements were performed using an inhouse setup built around a Karl Suss MicroTec PM300 waferprober, as illustrated in Fig. 2. The probe station was equipped
Table I. List of selected types of experimental PureGaB photodiodes, their alloying time, and Ge-island geometry, including on-mask values for the total area of Ge, lightentrance windows, and $\mathrm{Al}$ covering the contact-windows.

\begin{tabular}{llccllll}
\hline Diode name & $\begin{array}{l}\text { Alloy } \\
\text { time } \\
(\mathrm{min})\end{array}$ & $\begin{array}{l}\text { No. } \\
\text { of Ge } \\
\text { islands }\end{array}$ & $\begin{array}{l}\text { Ge-island } \\
\text { size }\left(\mu \mathrm{m}^{2}\right)\end{array}$ & $\begin{array}{l}\text { Total Ge } \\
\text { area size } \\
\left(\mu \mathrm{m}^{2}\right)\end{array}$ & $\begin{array}{l}\text { Entrance } \\
\text { window area } \\
\left(\mu \mathrm{m}^{2}\right)\end{array}$ & $\begin{array}{l}\text { Total Ge } \\
\text { perimeter } \\
(\mu \mathrm{m})\end{array}$ & $\begin{array}{l}\text { Al area on } \\
\text { contacts } \\
\left(\mu \mathrm{m}^{2}\right)\end{array}$ \\
\hline SPD26 & 30 & 1 & $26 \times 26$ & 676 & 576 & 104 & 153 \\
SPD12 & 30 & 4 & $12 \times 12$ & 576 & 400 & 192 & 529 \\
SPD5 & 30 & 16 & $5 \times 5$ & 400 & 144 & 320 & 585 \\
SPD50 & 30 & 1 & $11 \times 50$ & 550 & 432 & 122 & 180 \\
LPD26 & 60 & 1 & $26 \times 26$ & 676 & 576 & 104 & 153 \\
LPD12 & 60 & 4 & $12 \times 12$ & 576 & 400 & 192 & 329 \\
LPD5 & 60 & 16 & $5 \times 5$ & 400 & 144 & 320 & 585 \\
\hline
\end{tabular}




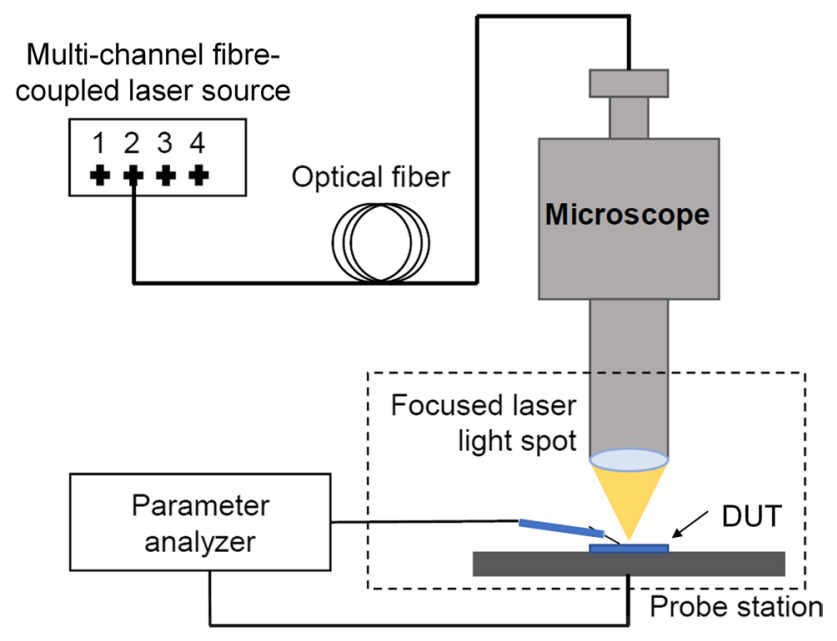

Fig. 2. Schematic of the optoelectrical measurement setup.

with an ATT liquid-cooled thermal chuck, allowing measurements in the temperature range $-50^{\circ} \mathrm{C}$ to $100^{\circ} \mathrm{C}$. In all cases, a Keithley 4200 parameter analyzer was used to measure the $I-V$ characteristics of the diodes under test (DUTs).

The setup was designed for on-wafer biasing of the DUT while it was exposed to laser light from a 4-channel laser source, Thorlabs MCLS1, using fiber-coupled laser diodes. The available wavelengths were $\lambda=406 \mathrm{~nm}, 670 \mathrm{~nm}, 1310$ $\mathrm{nm}$, and $1550 \mathrm{~nm}$. The fibre was connected to the lamp adapter of the probe station, and a spot size of approximately $10 \mu \mathrm{m}$ was focused on the DUT.

The transmission coefficient, $T_{\mathrm{c}}$, of the microscope was influenced by varying environmental factors. Therefore, a reference photocurrent was established by exposing a calibrated Thorlabs germanium photodetector to our light source, either directly from the fiber or by passing it through the optical path of the microscope setup. The corresponding photocurrents, $I_{\text {direct }}$ and $I_{\text {mscope }}$, respectively, were then used to calculate $T_{\mathrm{c}}$ as:

$T_{\mathrm{c}}=\frac{I_{\mathrm{mscope}}}{I_{\text {direct }}}$.

The optical power, $P_{\mathrm{DUT}}$, arriving at the DUT was then calculated as:

$P_{\text {DUT }}=P_{0} T_{\mathrm{c}}$

where $P_{0}$ is the optical power delivered by the laser. The $T_{\mathrm{c}}$ was monitored after each measurement of the diode current, $I_{\text {DUT }}$, of the PureGaB DUTs, giving the responsivity, $R$, as:

$R=\frac{I_{\mathrm{PD}}}{P_{\mathrm{DUT}}} ; I_{\mathrm{PD}}=I_{\mathrm{DUT}}-I_{\mathrm{dark}}$

where $I_{\text {dark }}$ is the dark current and $I_{\mathrm{PD}}$ the photogenerated current. It was possible to position the light spot within the light entrance window of diodes with sizes from $12 \times 12$ $\mu \mathrm{m}^{2}$ to $26 \times 26 \mu \mathrm{m}^{2}$. Most measurements were performed with a laser power of $1 \mathrm{~mW}$, which mainly corresponded to a power in the $10-20 \mu \mathrm{W}$ range landing on the DUT.

\section{Simulation Parameters}

Optoelectronic device simulations were performed using technology computer-aided design software, Synopsys Sentaurus Device. ${ }^{37}$ A Ge-on-Si structure was assumed with a thickness of the simulated $n$-type Si substrate of $500 \mu \mathrm{m}$ with constant doping, $N_{\mathrm{Si}}$, of $10^{15} \mathrm{~cm}^{-3}$. Simulated parameters of the Ge-island were extracted from the HRTEM analysis of the SPD diodes, i.e., a Ge thickness $t_{\mathrm{Ge}}=0.5 \mu \mathrm{m}$, and an oxide thickness $t_{\mathrm{ox}}=0.75 \mu \mathrm{m}$ were assumed. The $n$-type doping of the $\mathrm{Ge}$ island was constant, with concentration $N_{\mathrm{Ge}},=10^{16} \mathrm{~cm}^{-3}$. Since the optical properties of the Pure$\mathrm{GaB}$ layer were unknown, the PureGaB layer was omitted from the simulations, and instead a highly-doped $p^{+}$-region was assumed. Some Ge doping with $\mathrm{Ga}$ is expected during the $700^{\circ} \mathrm{C} 10$-min-long B-deposition but the resulting profile is not known. Due to the capping layer of B and the previously measured optical response for wavelengths as low as $255 \mathrm{~nm},{ }^{5}$ we suspect that the Ga doping is shallow and without roll-off at the Ge surface. Just as has been shown for PureB Si diodes, ${ }^{7}$ the reduction in responsivity can be quite low because the whole $p$-doping gradient effectively repels electrons from the surface. Therefore, to get a reasonable fit, we choose a 20 -nm-thick $p$-doping of the Ge surface with a constant doping of $10^{20} \mathrm{~cm}^{-3}$.

Fermi-Dirac statistics ${ }^{38}$ and carrier mobility using the Philips unified mobility mode ${ }^{39}$ were assumed for electrons and holes. The Shockley-Read-Hall generation-recombination model was used with electron and hole lifetimes for Si set to their default values. Based on, ${ }^{40}$ we assumed electron and hole lifetimes to be equal and for simplicity to have a value of $\tau_{\mathrm{e}}=\tau_{\mathrm{h}}=\tau_{\mathrm{e}, \mathrm{h}}=10^{-5} \mathrm{~s}$. Responsivity was extracted based on Eq. (3) by performing simulations without optical generation to obtain dark current values. Optical generation was modeled using the transfer matrix method model $^{37}$ which takes into account absorption, reflectivity, and interference in the simulated stack. Irrespective of the wavelength, an internal quantum yield of 1 was assumed.

\section{Results}

\section{Imaging Analysis}

In Fig. 3, HRTEM images of SPD devices are shown. The original shape of the as-grown Ge-island can be discerned but the processing of the contacts at the island perimeter has led to irregularities in the contours of the island. The oxide 


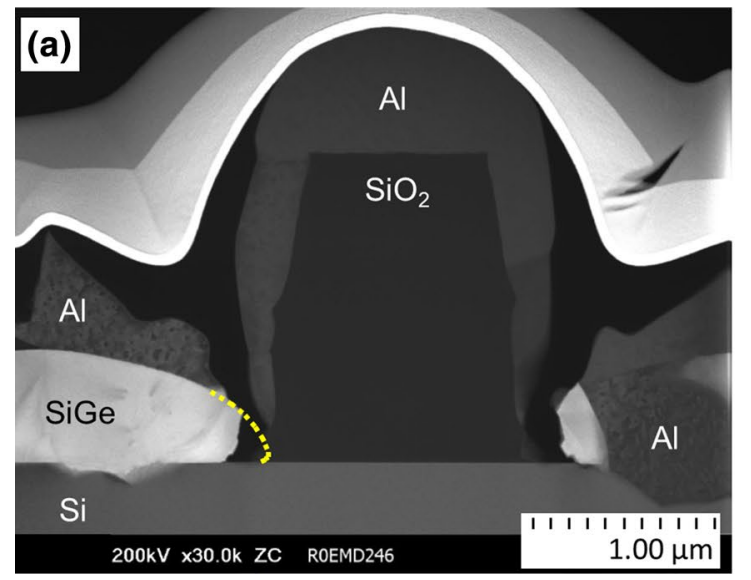

Fig. 3. HRTEM images of SPD diodes: (a) two Ge islands separated by $1 \mu \mathrm{m} \mathrm{SiO}{ }_{2}$, with a reconstruction (dotted yellow line) of where the tip of the Ge-island was removed because the Ge was not covered by

hills that separate the Ge-islands have sidewalls with several notches that were formed by the sequence of wet/plasma etch steps. When opening the windows for Ge deposition, a significant notch was formed at the base due to the softlanding plasma-etch step, and it is further enlarged by the pre-Ge-deposition HF dip. The Ge growth fills this notch, giving the pointed shape at the island edge, the extremity of which was thus covered by oxide when the Ga-B layer stack was deposited.

The etching of the window to contact the B-layer resulted in an opening larger than anticipated, exposing about $100 \mathrm{~nm}$ of the Ge-island. At this point, the $\mathrm{Al}$ reacted with the Ge, dissolving the Ge tip. In addition, Al-mediated transport of both $\mathrm{Ge}$ and $\mathrm{Si}$ is seen at the $\mathrm{Si}-\mathrm{Ge}$ interface where pitting of the $\mathrm{Si}$ appears up to $5 \mu \mathrm{m}$ away from the point of $\mathrm{Al}$ contact. At a few places, $\mathrm{Al}$ has coalesced into crystals. Apart from the point of $\mathrm{Al}$ contact, the top surface of the Ge-island is not deformed.

This is to be expected where it is covered with the 750-nm-thick oxide, but also, where the oxide has been replaced by $\mathrm{Al}$, the Ge surface only displays a very slight roughening that can be correlated to the underlying changes in the Ge crystal structuring. This is a confirmation that the B-layer functions as an efficient material barrier between the $\mathrm{Ge}$ and $\mathrm{Al}$.

In-line optical microscopy inspections of the diodes directly after metal patterning revealed no abnormalities of the $\mathrm{Al}$ patterns. However, after the alloying step, the effects of the Al-induced material transport were evident. An example is shown in Fig. 4 where microscopy images of the LPD26, LPD12, and LPD50 diodes are shown. The metal has moved away from the contact-window edge in an irregular fashion, with more metal appearing at the position where the tracks contact the diodes. This is particularly clear oxide; (b) and (c) a Ge-island where the $\mathrm{SiGe}$ and $\mathrm{Al}$ regions resulting from Al-induced material migration are visible, as indicated in (b) (Color figure online).

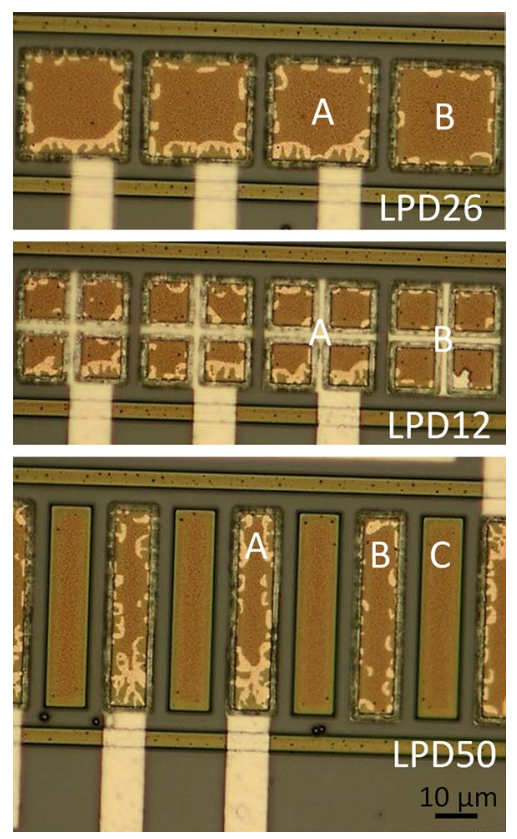

Fig. 4. Microscope images of the diodes LPD26, LPD12, and LPD50. The type A and B Ge-islands were processed as shown in Fig. 1, but A was connected to a metal track while B was not. The type C Ge-island remained covered with oxide and the metal on it was completely removed.

when comparing the diodes marked A and B that, respectively, do and do not have connections to a track. The diodes marked $\mathrm{C}$ are completely covered with oxide and all metal was removed.

Dies of SPD devices were given extra anneals. In Fig. 5, the result of a $500^{\circ} \mathrm{C} 30$-min anneal is shown. The $\mathrm{Al}$ structures that are not connected to any Ge-islands are not visibly affected by this temperature step, while those that are 
(a)

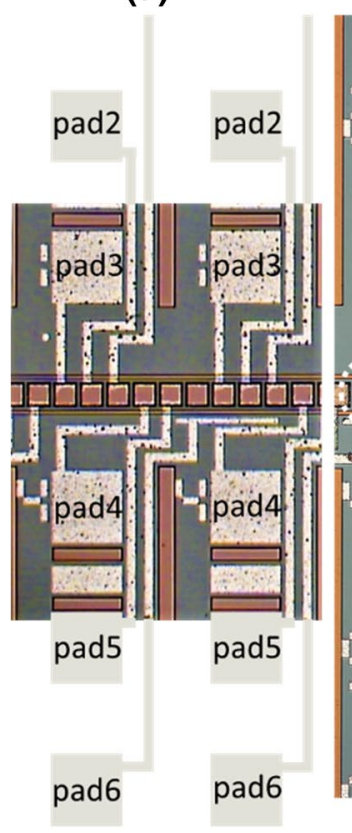

(b)

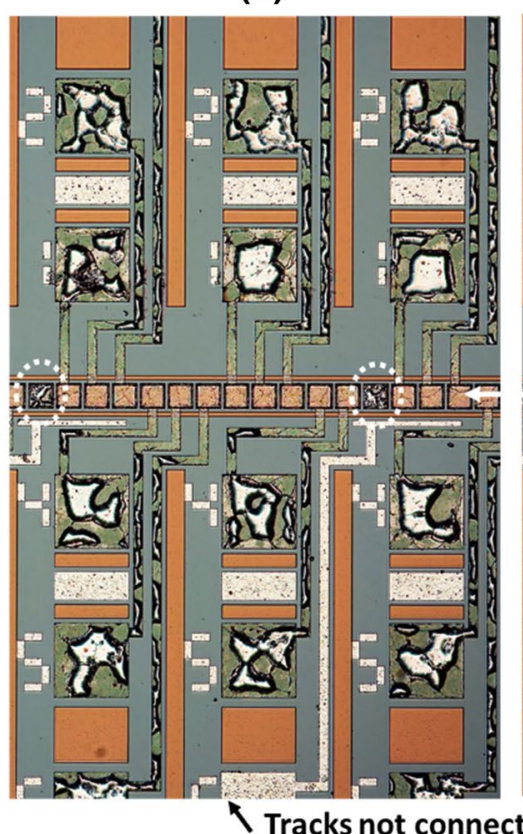

(c)

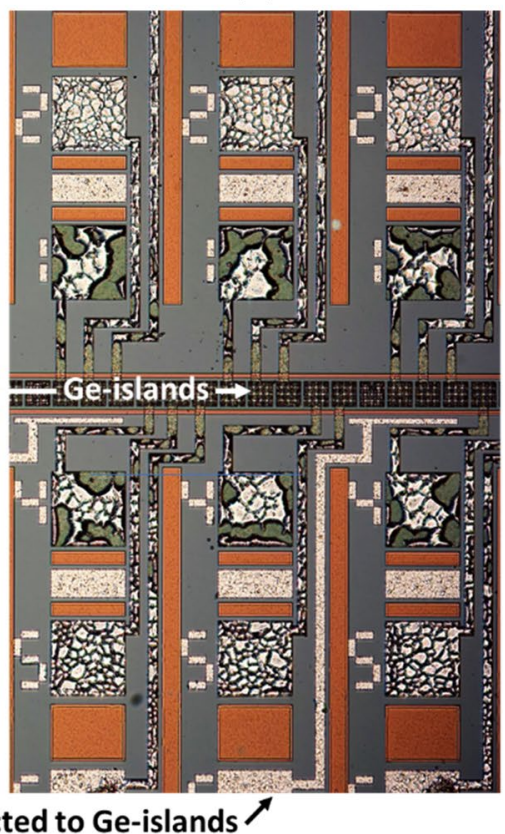

Fig. 5. Microscope images of a row of (a) non-annealed LPD26 diodes combined with a schematic of the measurement pad/track layout, and rows of (b) SPD26 diodes and (c) SPD5 diodes (composed of a $4 \times 4$ array of $5 \times 5 \mathrm{um}^{2}$ diodes), both annealed at $500^{\circ} \mathrm{C}$ for 30

connected, including both tracks and pads, are seriously deformed. The $\mathrm{Al}$ seems to disappear on and near the $\mathrm{Ge}$ islands, coalescing into droplets of $\mathrm{Al}$ along the rest of the tracks and on the pads.

The movement of the Al to form droplets was confirmed by AFM imaging of one of the affected pads, as shown in Fig. 6. The height profiles confirm that the Al has disappeared in some places and is about double the nominal thickness in other places, whereas the metal that is not connected to a Ge-island has the nominal thickness of $\sim 750$ min. Two SPD26 diodes that are not connected to a metal track are circled by white dashed lines. Only every second pad 6 is connected to a Ge-island.

$\mathrm{nm}$. Although the metal tracks were seriously deranged, the electrical contact was preserved due to remnants of $\mathrm{Al}$ that were particularly visible where the original grain boundaries appeared to have been. Hence, the optoelectrical measurements could also be made on these diodes.

Of particular interest was the behavior at temperatures that could occur during device operation. Therefore, SPD devices were exposed to several anneals at $100^{\circ} \mathrm{C}$, one with a total time of $40 \mathrm{~h}$. The microscopy images of the SPD26 and SPD50 diodes are shown in Fig. 7. The metal patterns
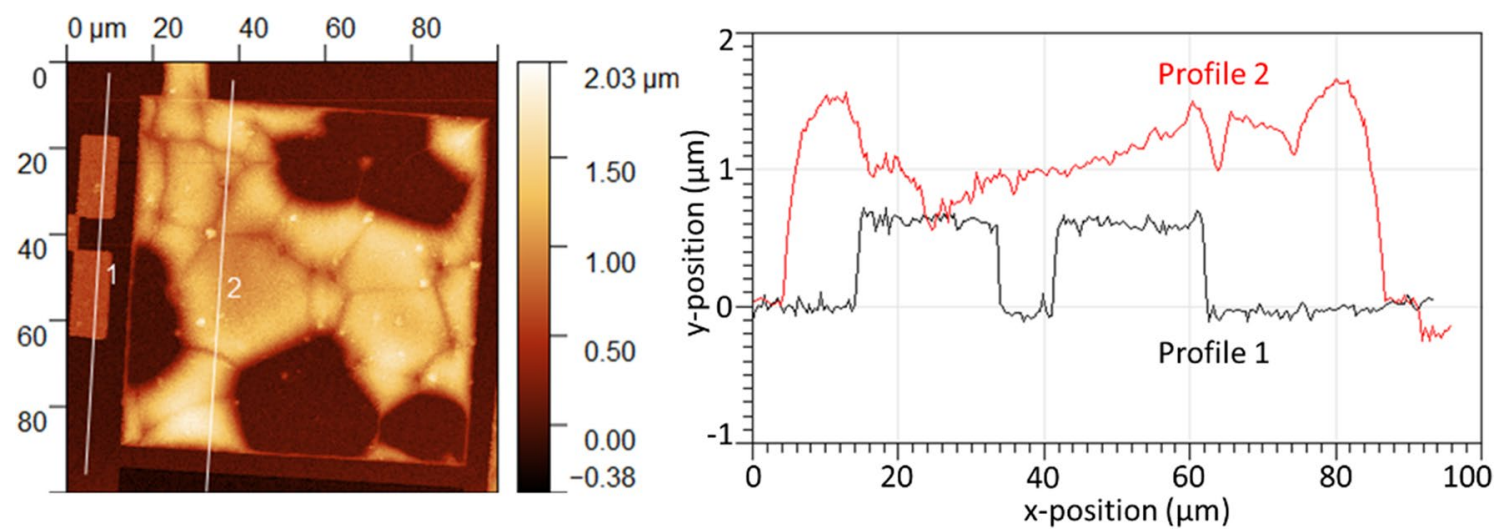

Fig. 6. AFM image and height profile of metal patterned on the oxide only (Profile 1) and metal in a measurement pad connected to a SPD12 diode after annealing at $500^{\circ} \mathrm{C}$ for $30 \mathrm{~min}$ (Profile 2). 
before and after annealing are identical. In addition, it can be noted that the amount of displaced $\mathrm{Al}$ is much less than for the LPD diodes of Fig. 5 that had a 30 -min longer $400^{\circ} \mathrm{C}$ alloy step.

\section{Electrical Characterization}

In Fig. 8, the $I-V$ characteristics of the diodes are plotted along with those of a comparable Si PureB diode of similar size fabricated on an $n$-Si substrate with doping $\sim 10^{15} \mathrm{~cm}^{-3}$. The current of the Si diode is more than 3 decades lower than the Ge diodes, due to the much higher Si bandgap of $1.12 \mathrm{eV}$ as compared to $0.66 \mathrm{eV}$ for Ge. The 3 different $\mathrm{Ge}$ diodes have current differences that roughly follow the differences in Ge-island area. For the LPD diodes the current differences are also present but they are much smaller and not shown here.

In Fig. 9, the effect of the $100^{\circ} \mathrm{C} 40$-h anneal on the $I-V$ characteristics of the SPD50 diodes can be seen. As for the imaging analysis, no modification of the original situation is visible. Several curves measured after the $500^{\circ} \mathrm{C}$ 30-min anneal are also shown. In this case, the current was reduced by a few factors and the spread in current increased considerably.

Temperature-dependent measurements from $-50^{\circ} \mathrm{C}$ to $100^{\circ} \mathrm{C}$ were also performed on a large number of both SPD and LPD diodes of different sizes, with and without the extra anneals. Richardson plots of $\ln \left(I / T^{2}\right)$ as a function of $1 / T$ for each measured diode were made from the $I-V$ characteristics to extract the bandgaps ${ }^{38}$ and they all fell in the range $0.66 \mathrm{eV}$ to $0.75 \mathrm{eV}$ but with quite some spread even across
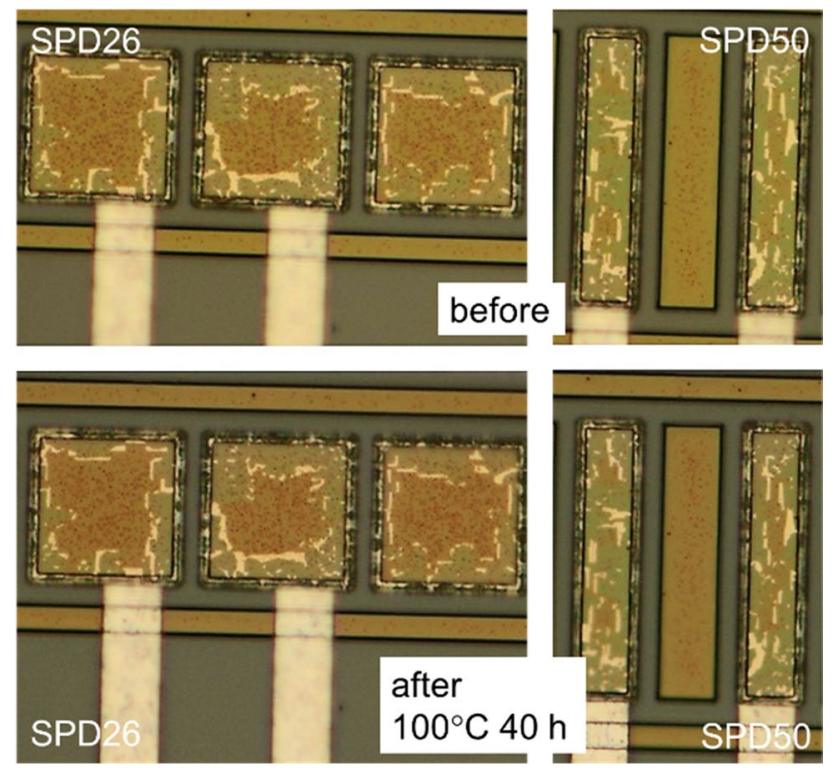

Fig. 7. Microscope images of SPD26 and SPD50 diodes before and after a $100^{\circ} \mathrm{C}$ anneal for $40 \mathrm{~h}$.

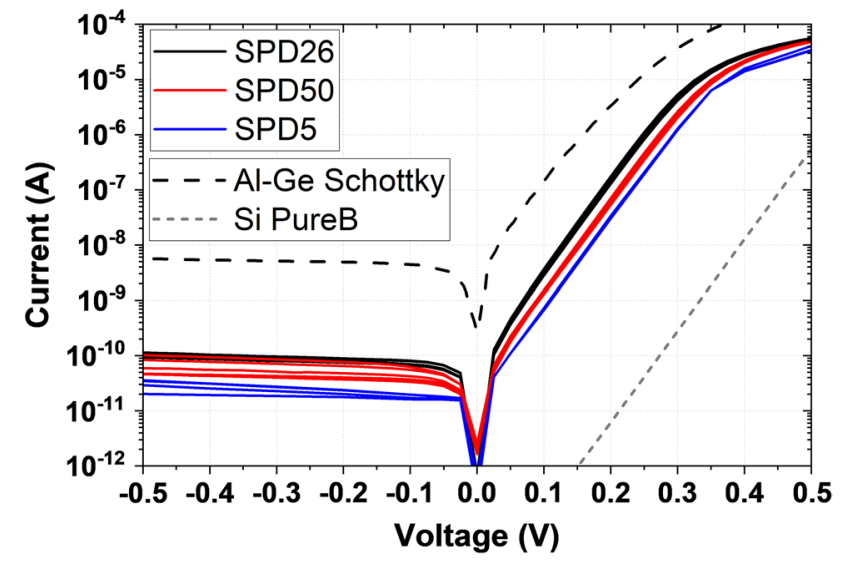

Fig. 8. Measured $I-V$ characteristics for several SPD26, SPD50, and SPD5 diodes compared to those of a Si PureB diode, fabricated as described in Ref. 41 , and an Al-Ge Schottky diode, ${ }^{42}$ both of size $40 \times 40 \mu \mathrm{m}^{2}$ and processed on a substrate with doping $10^{15} \mathrm{~cm}^{-3}$.

neighboring diodes of the same type, and no clear trends were found. This can be related to the presence of small but variable non-ideal currents which become visible in the reverse characteristics of the diodes as seen in Figs. 8 and 9. Nevertheless, it can be concluded that the bandgaps are close to the characteristic Ge values in all cases.

\section{Optical Characterization}

The $I-V$ characteristics of an SPD26 diode are shown in Fig. 10 for illumination with an output power of $1 \mathrm{~mW}$ for each of the lasers. For all the 4 wavelengths, the lightinduced current is decades higher than the dark current. In Fig. 11, the extracted responsivity at each wavelength for a number of SPD26, SPD12, LPD26, and LPD12 diodes is

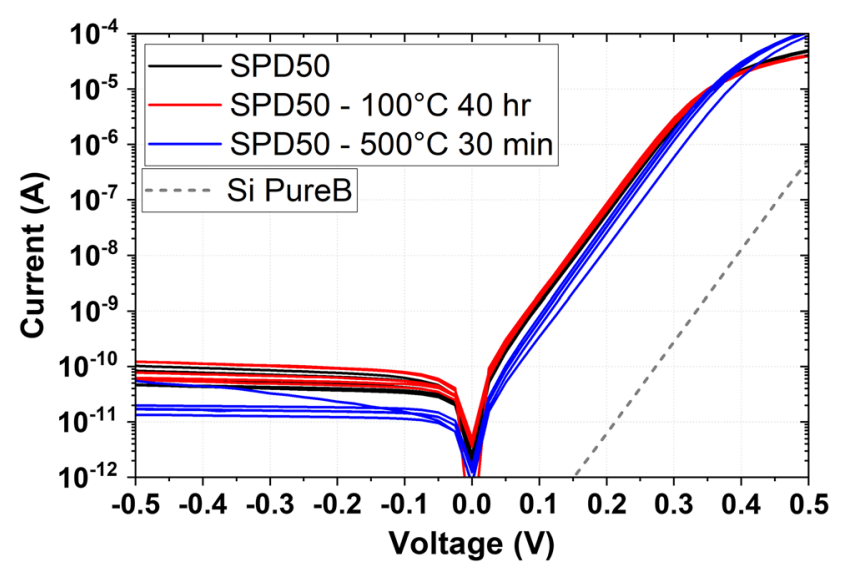

Fig. 9. Measured $I-V$ characteristics for several SPD50 diodes before and after anneals at $100^{\circ} \mathrm{C}$ for $40 \mathrm{~h}$ and at $500^{\circ} \mathrm{C}$ for $30 \mathrm{~min}$, compared to those of a Si PureB diode of size $40 \times 40 \mu \mathrm{m}^{2}$ processed on a substrate with doping $10^{15} \mathrm{~cm}^{-3}$, fabricated as described in Ref. 41 . 


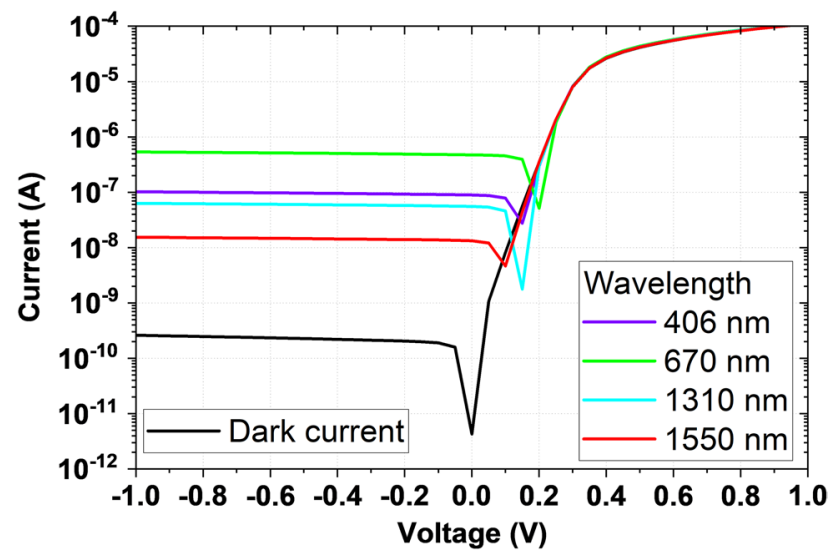

Fig. 10. Measured $I-V$ characteristics of an SPD26 diode with and without illumination with a $1-\mathrm{mW}$ output power of the lasers, for wavelengths $406 \mathrm{~nm}, 670 \mathrm{~nm}, 1310 \mathrm{~nm}$, and $1550 \mathrm{~nm}$.

plotted and compared to the simulated responsivity. The best correspondence with the simulated values is for the SPD26 device at the short wavelengths of $406 \mathrm{~nm}$ and $670 \mathrm{~nm}$. At $1310 \mathrm{~nm}$ and $1550 \mathrm{~nm}$, the responsivity of this diode is still significant, but 5-10 times lower than the simulated values. The SPD12 diode has similar values as the SPD26 diode, but the responsivity is repeatedly a little bit lower. Since the light-entrance window on the individual Ge-islands of this composite diode is about the same size as the light spot, there is a reasonable chance that misalignment errors are playing a role. In all cases, the LPD diodes have much lower responsivity than the SPD diodes, about 5 times lower for the LPD26 diodes. However, at $1310 \mathrm{~nm}$ and $1550 \mathrm{~nm}$, the discrepancy between the LPD26 and LPD12 diodes

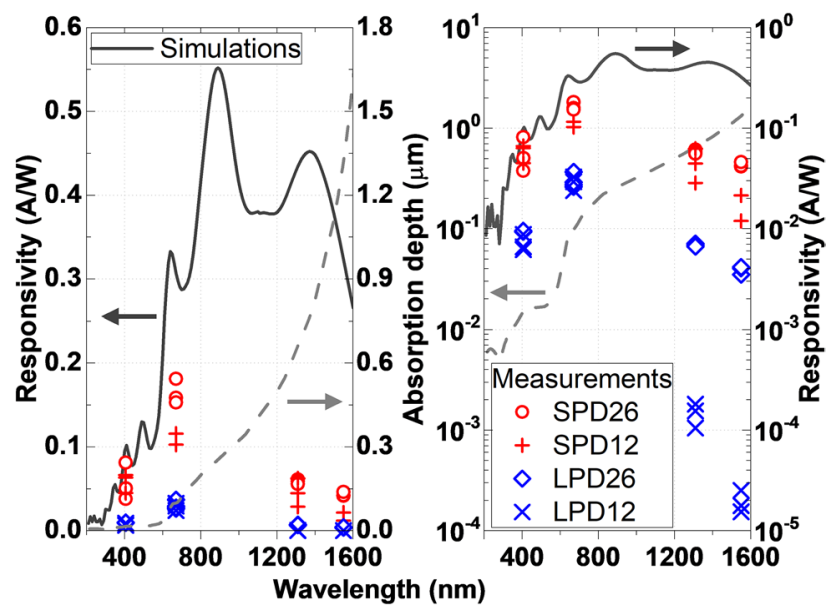

Fig. 11. Simulated responsivity (solid line) and extracted absorption depth (dashed line) as a function of wavelength compared to responsivity values extracted from optical measurements of SPD26, SPD12, LPD26, and LPD12 diodes. becomes so large, more than a decade, that it can no longer be accorded to misalignment errors.

In Fig. 12, the responsivity after $100^{\circ} \mathrm{C}$ and $500^{\circ} \mathrm{C}$ anneals is plotted for a number of SPD26 diodes. As was the case for the $I-V$ characteristics, the $100^{\circ} \mathrm{C}$ anneal, even though it was performed for tens of hours, had no significant effect. On the other hand, the $500^{\circ} \mathrm{C} 30$-min anneal gave a significant degradation of the responsivity at all wavelengths. At $406 \mathrm{~nm}$ and $670 \mathrm{~nm}$, there is about a 70\% decrease, while the responsivity has all but disappeared at $1310 \mathrm{~nm}$ and $1550 \mathrm{~nm}$.

\section{Discussion}

The Ge-island regions affected by the Al-mediated material transport along the Ge-Si interface become less light sensitive for all wavelengths. The responsivity of the SPD diodes in the central anode region that is untouched by the detrimental perimeter effects is almost ideal for the visual wavelengths that are predominantly collected near the Ge surface. In contrast, the responsivity at the SWIR wavelengths is much lower. As was demonstrated via simulations in Ref. ${ }^{5}$, this can be due to defects at the Ge-Si interface that trap the light-generated carriers. Combined with the fact that the Ge-island thickness is about half the absorption length of the near-infrared (NIR) wavelengths, this can account for the low responsivity. Electrically, the presence of the interface defects is seen as a rise in the reverse leakage current when the depletion region transverses the interface. This occurs at about $6 \mathrm{~V}$ reverse bias in the present devices.

The Ge-Si interface and the associated crystal defects are also important for the progression of the $\mathrm{Al}$ migration. If $\mathrm{Al}$ was deposited on a planar crystalline Ge surface, annealing around $400^{\circ} \mathrm{C}$ would cause localized spiking of the Ge surface with inverted pyramids, a process that normally would

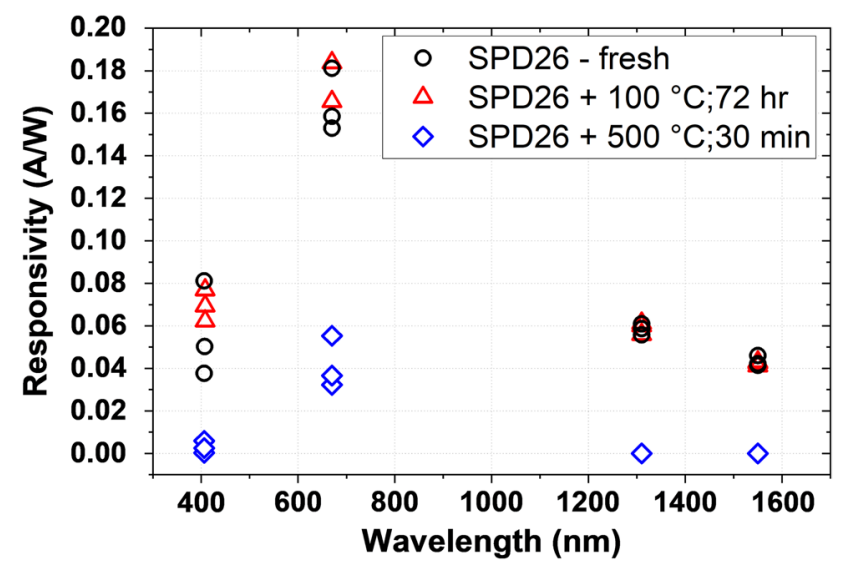

Fig. 12. Responsivity of SPD26 diodes as a function of wavelength, with and without anneals at $100^{\circ} \mathrm{C}$ for $72 \mathrm{~h}$ and $500^{\circ} \mathrm{C}$ for $30 \mathrm{~min}$. 
stop when the $\mathrm{Al}$ is saturated with $\mathrm{Ge}$, as determined by $\mathrm{Ge}-\mathrm{Al}$ eutectic system. ${ }^{30} \mathrm{In}$ the present devices, the role of Ge saturation is particularly clear after the $500^{\circ} \mathrm{C}$ anneal, at which temperature the alloying with Ge liquifies the $\mathrm{Al}$ tracks. The $\mathrm{Al}$ then coalesces into droplets leaving large areas practically cleared of Al, particularly on and near the $\mathrm{Ge}$-islands. The distance over which such $\mathrm{Al}$ displacement is seen decreases when the ratio of the total Ge diode area to the area of $\mathrm{Al}$ at the contacts decreases. An example is seen in Fig. 5 where images are shown of the tracks leading to SPD26 and SPD5 diodes. From the areas listed in Table I, the ratio is $676 / 153=4.4$ for the SPD26 diodes and 400/585 $=0.7$ for the SPD5 diodes. This large difference has meant that the most distant pad (pad 6) is only mildly affected for the SPD5 diodes, while it is heavily deranged for SPD26 diodes.

In the present diodes, even the SPDs, the $\mathrm{Al}$ dissolved enough $\mathrm{Ge}$ to reach the Ge-Si interface, which appears to have enhanced the material transport possibilities, resulting in pitting of the Si. From the TEM images in Fig. 3, it is clear that this pitting does not necessarily occur immediately at the small, $\sim 0.1-\mu \mathrm{m}$-wide, Al point-of-contact with the $\mathrm{Si}$. Most likely, the pitting is initiated at specific interface defects, perhaps stress-related, that remain after the annealing of the Ge-island at $750^{\circ} \mathrm{C}$. The Si pits are filled with Ge that is distinguished by being a darker shade of gray, as indicated in Fig. 3b. These dark gray regions extend to the $\mathrm{Ge}$ surface, being bounded by a network of misfit dislocations that suggests an $\mathrm{Al}$-mediated rearrangement of the original crystal structure. The surrounding light gray regions display fewer dislocations. The Si content of these two regions was monitored by EDS, as reported in Ref. ${ }^{1}$,showing no measurable $\mathrm{Si}$ in the light gray regions and low but significant amounts in the dark gray regions. In some places, the $\mathrm{Al}$ is also seen to have coalesced into grains that were measured to have only $\mathrm{Al}$ content. These grains only appear in connection with pitted Si regions, and plausibly mark the end of the $\mathrm{Al}$ migration at the given temperature because they would be saturated with Ge and/or Si.

The EDS analysis did not reveal any $\mathrm{Al}$ in the gray $\mathrm{Ge}$ regions, but it is safe to assume that there is an $\mathrm{Al}$ doping of the affected regions to a level that makes them $p$-type material. Although we have found no other studies of our particular material-exchange situation, several layerexchange studies, using a layer stack of amorphous $\mathrm{Ge}$ and $\mathrm{Al}$ on crystalline $\mathrm{Si}$, suggest that a $\mathrm{Si}_{x} \mathrm{Ge}_{x-1}$ alloy would be formed with a tapering off of the Si concentration towards the surface of the Ge crystal. ${ }^{31,32}$ One paper on Ge/Al layerexchange at $350^{\circ} \mathrm{C}$ reports solid-phase-epitaxy (SPE) of Ge doped with $\mathrm{Al}$ to as much as $10^{17} \mathrm{~cm}^{-3} .33,34$

To our knowledge, the literature does not report any values for the active $\mathrm{Al}$ dopant concentration in such $\mathrm{Ge}$ SPE layers. For Si, studies of layer-exchange Al-doped crystalline Si layers formed at $400^{\circ} \mathrm{C}$, using secondary-ion mass spectrometry and electrical measurements, estimated an active doping level in the $10^{18} \mathrm{~cm}^{-3}$ range ${ }^{43}$ and with this $p$-material, $p^{+} n$-type junctions were formed on $n$-Si. ${ }^{44} \mathrm{In}$ $\mathrm{Ge}$, a higher active $\mathrm{Al}$ doping could be expected due to the very high solid solubilities above $10^{20} \mathrm{~cm}^{-3}$ at temperatures around $400^{\circ} \mathrm{C}^{45}$ There are also reports in the literature that give reason to believe that there will be an increased solubility of $\mathrm{Si}$ in $\mathrm{Al}$ due to the presence of $\mathrm{Ge}$, and that the $\mathrm{Al}$ liquification temperature may also be lowered due to interactions with both these materials. ${ }^{46,47}$

The $p$-doping of the Ge above the Si pits can explain both the electrical and optical behavior of the diodes. While the anneal-induced changes in responsivity were large, the $I-V$ characteristics showed only moderate changes. This can be correlated to the increasing shift of the $p n$ Ge junction towards a ( $p-\mathrm{Ge})$-on- $(n-\mathrm{Si})$ junction. For both $\mathrm{Ge}-\mathrm{Ge}$ and $\mathrm{Ge}-\mathrm{Si} p n$ junctions on the $\mathrm{Si}$ substrate, the electron injection into the Ge, governed by the small Ge bandgap, will remain large. As opposed to this, the hole injection is only slightly reduced when the $n$-Ge is replaced by $n$-Si. For Si $p n$ junctions to the $n$-substrates used here, the current is dominated by hole injection into the substrate, and this is decades lower than the SPD current levels, as can be seen from the Si diode $I-V$ characteristic included in Fig. 8. Upon $500^{\circ} \mathrm{C}$ annealing, the current of the SPD diodes was only lowered by a factor of about 2-5. This spread is perhaps related to the presence of Ge-Si alloys that would give some increase in the $p$-Ge bandgap, but the extraction of the diode bandgaps was not accurate enough to substantiate something like that.

It is noteworthy that the differences in current levels between the different-sized SPD diodes roughly follow the size of the total Ge-island. Ge-islands that were contacted directly by Al displayed Schottky junction characteristics with 2-3 decades higher saturation currents than the PureGaB counterparts, ${ }^{42}$ as shown in Fig. 8. Although the Ge-island perimeter in the SPD and LPD diodes was inadvertently contacted directly by $\mathrm{Al}$, the current levels are not higher than what is expected for PureGaB diodes. This suggests that the perimeter remains $p$-type, either due to sufficient Ga-doping or as a result of the anomalous material exchange processes. When the latter does result in a $p$-doping of the sidewalls, this would have the advantage of improving the passivation of the interface with the dielectric by moving the depletion across the $p n$-junction away from interface defects.

The LPD diodes have a 30-min longer $400^{\circ} \mathrm{C}$ anneal which is seen in Fig. 11 to have a negative impact on the responsivity. There is about a factor 8 decrease in the responsivity for all wavelengths, showing that the central diode regions were also impacted by the material transport for this longer alloy time. However, a difference between the LPD26 and LPD12 diodes is only seen for the NIR wavelengths, for 
which the LPD12 diodes have about 2 decades lower responsivity, thus all but disappearing. This can be accorded to changes at the interface that reduce NIR responsivity, while the shorter wavelengths that are detected predominantly at the Ge surface are much less affected. This suggests that the migration of $\mathrm{Al}$ along the interface progressed much faster than the mechanisms leading to the modified Gecrystals, alloyed with $\mathrm{Si}$ and Al-doped, that span the full Ge thickness.

Since the low NIR responsivity for the SPD and LPD diodes is correlated to the conditions at the Ge-Si interface, in this wavelength range, it is obviously an advantage to have much thicker Ge-islands than the ones studied here. For broadband applications, the Al-induced perimeter effects would then be acceptable in the situation where the Geisland is large with respect to the affected perimeter width. The $100^{\circ} \mathrm{C}$ anneals of the SPD diodes were therefore performed to assess whether the perimeter could be expected to degrade under normal linear mode photodiode operation. Neither the $I-V$ nor the responsivity showed any significant change even after a total of $72 \mathrm{~h}$ annealing. The operation of LPD diodes as APDs, which could cause material migration due to the high electric fields over the Ge junctions, was also studied in Ref. ${ }^{2}$. Repeated measurements did not lead to an attenuation of the optoelectronic characteristics being monitored. However, more long-term stability testing would be needed to ascertain the true value of this result.

Poorly-controlled mechanisms, like the Al-mediated material migration described here, should mainly be avoided if only for reliability and reproducibility reasons. For PureGaB Ge-on-Si photodiodes a few microns in size, there is also a trade-off to be considered between fill-factor and series resistance/process complexity. Ideally, the metal contacting should be made as narrow as possible along the anode perimeter region but this is with the risk, documented here, of removing the protective $\mathrm{B}$ material barrier at the $\mathrm{Ge}$ edge. Increasing reliability by making the B-layer thicker is not always an option because tunneling-layer thickness, i.e., less than about $3 \mathrm{~nm}$, is needed to assure low contact resistance. ${ }^{27}$ Therefore, the direct $\mathrm{Al}$ contacting of the Ge as realized in the SPD diodes could be seen as a viable, lowcost option in some situations, since there is no trade-off in dark current. Another "safe" option would be to deliberately remove the B-layer in the contact windows and, at the cost of increasing process complexity, deposit a new well-defined Ga-B layer stack before the metal deposition.

\section{Conclusions}

An anomalous Al-mediated material-transport process was assessed with respect to the applicability of PureGaB Geon-Si diodes as broadband photodiodes. The theoretical responsivity found from simulations was also measured for $406 \mathrm{~nm}$ and $670 \mathrm{~nm}$ light, confirming the high surface sensitivity of the ultrashallow PureGaB Ge diodes. In contrast, the responsivity at $1310 \mathrm{~nm}$ and $1550 \mathrm{~nm}$, that could have been achieved with the modest Ge-island thickness of $0.5 \mu \mathrm{m}$, was far from being reached due to the proximity of the Ge-Si interface, which introduced a high density of crystal defects in the main light-absorbing region of these wavelengths.

These interfacial defects also played a decisive role in the Al-induced material transport. The B-layer of the PureGaB anode prevented reactions with the Ge, but, in our experiments, at the $\mathrm{Ge}$-island perimeter, the $\mathrm{Al}$ was able to reach the $\mathrm{Si}$ after which it traveled along the $\mathrm{Ge}-\mathrm{Si}$ interface where localized Si pits formed. These pits were filled with Ge crystals alloyed with $\mathrm{Si}$ and doped with Al. After the standard $30-\min 400^{\circ} \mathrm{C}$ anneal, such pitting was observed up to about $5 \mu \mathrm{m}$ from the perimeter. Longer/higher temperature treatment in the $400-500^{\circ} \mathrm{C}$ range aggravated this situation, greatly affecting the NIR and ultimately also extinguishing the visual responsivity. This was found to be due to an increased $\mathrm{Al} p$-doping of the Ge that shifted the $p n$ metallurgic junction from the bulk Ge to the Ge-Si interface.

The NIR responsivity can be increased by growing thicker Ge-islands, something that is generally done. This would also reduce the disadvantage for the responsivity posed by the anomalous p-doping with Al. It is also noteworthy that, after the standard alloying step, the dark currents were low, and they were not degraded by any of the extra heat treatments. In addition, neither the responsivity nor the dark current changed after prolonged annealing at $100^{\circ} \mathrm{C}$, which represents a maximum for temperatures that may occur during normal device operation. Therefore, for some PureGaB photodiode configurations, the direct $\mathrm{Al}$ contacting of $\mathrm{Ge}-$ island sidewalls, may be a low-cost method of achieving both low contact resistance to the anode, $p$-type sidewall passivation, and a maximal fill-factor.

Acknowledgments The authors would like to thank Amir Sammak for the device processing in the former DIMES-ICP processing cleanroom and for the previously documented analysis of the results. This work was supported by the Croatian Science Foundation under the Project IP-2018-01-5296 and the B-Power Project No. 17979 of The Netherlands Organization for Scientific Research (NWO) Domain Applied and Engineering Sciences (TTW).

Conflict of interest The authors declare that they have no known competing financial interests or personal relationships that could have appeared to influence the work reported in this paper.

Open Access This article is licensed under a Creative Commons Attribution 4.0 International License, which permits use, sharing, adaptation, distribution and reproduction in any medium or format, as long as you give appropriate credit to the original author(s) and the source, provide a link to the Creative Commons licence, and indicate if changes were made. The images or other third party material in this article are 
included in the article's Creative Commons licence, unless indicated otherwise in a credit line to the material. If material is not included in the article's Creative Commons licence and your intended use is not permitted by statutory regulation or exceeds the permitted use, you will need to obtain permission directly from the copyright holder. To view a copy of this licence, visit http://creativecommons.org/licenses/by/4.0/.

\section{References}

1. A. Sammak, L. Qi, and L.K. Nanver, J. Electron. Mater. 44/12, 4676 (2015).

2. A. Sammak, M. Aminian, L.K. Nanver, and E. Charbon, IEEE Trans. Electron Devices 63/1, 92 (2016).

3. D.T. Shivakumar, T. Knežević, and L.K. Nanver, J. Mater. Sci. Mater. Electron. 32/6, 7123 (2021).

4. H. Kim, J. Vac. Sci. Technol. B 21, 2231 (2003).

5. T. Kneževic, M. Krakers, L.K. Nanver, Broadband PureGaB Geon-Si photodiodes responsive in the ultraviolet to near-infrared range, in Proceedings of SPIE 11276, Optical Components and Materials XVII (2020), p. 112760I.

6. L. Marković, T. Knežević, L.K. Nanver, T. Suligoj, Modeling and Simulation Study of Electrical Properties of Ge-on-Si Diodes with Nanometer-thin PureGaB Layer, in Proceedings of 44th International Convention on Information and Communication Technology, Electronics and Microelectronics (MIPRO) (2021).

7. L. Shi, S. Nihtianov, L. Haspeslagh, F. Scholze, A. Gottwald, and L.K. Nanver, IEEE Trans. Electron Devices 59/11, 2888 (2012).

8. E.D. Palik, Handbook of Optical Constants of Solids, Vol. 1 (New York: Academic, 1985).

9. W. Zheng, L. Jia, and F. Huang, iScience 23/6, 1 (2020).

10. D.E. Aspnes, and A.A. Studna, Phys. Rev. B 27/2, 985 (1983).

11. F.J. Wilkinson, A.J.D. Farmer, and J. Geist, J. Appl. Phys. 54/2, 1172 (1983).

12. E. Antoncik, and N.K.S. Gaur, J. Phys. C 11/4, 735 (1978).

13. A. Satta, E. Simoen, T. Janssens, T. Clarysse, B. De Jaeger, A. Benedetti, I. Hoflijk, B. Brijs, M. Meuris, and W. Vandervorst, J. Electrochem. Soc. 153/3, G229 (2006).

14. S.-S. Chuang, T.-C. Cho, P.-J. Sung, K.-H. Kao, H.J.H. Chen, Y.-J. Lee, M.I. Current, and T.-Y. Tsenga, ECS J. Solid State Sci. Technol. 6/5, P350 (2017).

15. J. Li, R. Cheng, C. Liu, P. Zhang, J. Lu, K. Chen, R. Zhang, and Y. Zhao, Microelectron. Eng. 168, 1 (2017).

16. J. Sharp, W.J. Lee, K. Ploog, G.A. Umana-Membreno, L. Faraone, and J.M. Dell, Solid-State Electron. 89, 146 (2013).

17. R.E. Warburton, G. Intermite, M. Myronov, P. Allred, D.R. Leadley, K. Gallacher, D.J. Paul, N.J. Pilgrim, L.J.M. Lever, Z. Ikonic, R.W. Kelsall, E. Huante-Cerón, A.P. Knights, and G.S. Buller, IEEE Trans. Electron Devices 60/11, 3807 (2013).

18. D. Benedikovic, L. Virot, G. Aubin, J.-M. Hartmann, F. Amar, X. Le Roux, C. Alonso-Ramos, É. Cassan, D. Marris-Morini, J.-M. Fédéli, F. Boeuf, B. Szelag, and L. Vivien, Nanophotonics 10/3, 1059 (2021)

19. A. Sammak, M. Aminian, L. Qi, W.B. de Boer, E. Charbon, L.K. Nanver, A CMOS compatible Ge-on-Si APD operating in proportional and Geiger modes at infrared wavelengths, in Proceedings of International Electron Devices Meeting (2011), p. 8.

20. Y. Kang, H.-D. Liu, M. Morse, M.J. Paniccia, M. Zadka, S. Litski, G. Sarid, A. Pauchard, Y.-H. Kuo, H.W. Chen, W.S. Zaoui, J.E. Bowers, A. Beling, D.C. McIntosh, X. Zheng, and J.C. Campbell, Nature Photon. 3/1, 59 (2009).

21. M. Takenaka, K. Morii, M. Sugiyama, Y. Nakano, and S. Takagi, Opt. Exp. 20/8, 8718 (2012).
22. I. Åberg, B. Ackland, J. V. Beach, C. Godek, R. Johnson, C.A. King, A. Lattes, J. O’Neill, S. Pappas, T.S. Sriram, and C.S. Rafferty, A Low Dark Current and High Quantum Efficiency Monolithic Germanium-on-Silicon CMOS Imager Technology for Day and Night Imaging Applications, in Proceedings of International Electron Devices Meeting (2010), p. 344.

23. P. Veis, A. Marín-Roldán, and J. Krištof, Plasma Sources Sci. Technol. 27/9, 095001 (2018).

24. C. Farley III, A. Kassu, S. Sadate-Moualeu, J. Mills, C. Brantley, E. Edwards, P. Ruffin, A. Sharma, Broadband optical sensing/ detection technology for missile systems, in Proceedings of SPIE 10746, Novel Optical Systems Design and Optimization XXI, (2018) p. 1074608.

25. S.M. Skippon, and R.T. Short, Fire Saf. J. 21/1, 1 (1993).

26. S. Goossens, G. Navickaite, C. Monasterio, S. Gupta, J.J. Piqueras, R. Pérez, G. Burwell, I. Nikitskiy, T. Lasanta, T. Galán, E. Puma, A. Centeno, A. Pesquera, A. Zurutuza, G. Konstantatos, and F. Koppens, Nat. Photonics 11/6, 366 (2017).

27. J. Jiang, J.H. Chu, and K. Banerjee, CMOS-Compatible DopedMultilayer-Graphene Interconnects for Next-Generation VLSI, in Proceedings of IEEE International Electron Devices Meeting (2018), p. 34.5.1.

28. J. Michel, J. Liu, and L.C. Kimerling, Nat. Photonics 4/8, 527 (2010).

29. J. Liu, R. Camacho-Aguilerab, J.T. Bessette, X. Sun, X. Wanga, Y. Cai, L.C. Kimerling, and J. Michel, Thin Solid Films 520/8, 3354 (2012).

30. A.J. McAlister, and J.L. Murray, Bull. Alloy Phase Diagr. 5/4, 341 (1984).

31. Z. Liu, X. Hao, F. Qi, A. Ho-Baillie, and M.A. Green, Scr. Mater. $71,25(2014)$

32. C.-J. Lin, S.-Y. Wei, C.-C. Hsu, S.-M. Yu, W.-C. Sun, T.-S. Lin, and F.-R. Chen, Cryst. Eng. Comm. 17/33, 6269 (2015).

33. T. Nishida, M. Nakata, T. Suemasu, and K. Toko, Thin Solid Films 681, 98 (2019).

34. Z.M. Wang, J.Y. Wang, L.P.H. Jeurgens, and E.J. Mittemeijer, Surf. Interface Anal. 40/3-4, 427 (2008).

35. A.F.M. Leenaars, J.A.M. Huethorst, and J.J. van Oekel, Langmuir 6/11, 1701 (1990).

36. A. Sammak, W.B. de Boer, and L.K. Nanver, ECS Trans. 50/9, 507 (2013).

37. Synopsys, Sentaurus Device User Guide Version N-2017.09. (Mountain View: Synopsys, 2017).

38. S.M. Sze, and K.K. Ng, Physics of Semiconductor Devices, 3rd ed., (Hoboken: Wiley, 2006).

39. D.B.M. Klaassen, Solid-State Electron. 35/7, 953 (1992).

40. E. Gaubas, and J. Vanhellemont, Appl. Phys. Lett. 89/14, 142106 (2006).

41. F. Sarubbi, L.K. Nanver, and T.L.M. Scholtes, IEEE Trans. Electron Devices 57/6, 1269 (2010).

42. A. Sammak, L. Qi, W.B. de Boer, and L.K. Nanver, Solid-State Electron. 74, 126 (2012).

43. Y. Civale, L.K. Nanver, S.G. Alberici, A. Gammon, and I. Kelly, Electrochem. Solid-State Lett. 11/4, H74 (2008).

44. Y. Civale, L.K. Nanver, P. Hadley, E.J.G. Goudena, and H. Schellevis, IEEE Electron Device Lett. 27/5, 341 (2006).

45. F.A. Trumbore, Bell Syst. Tech. J. 39/1, 205 (1960).

46. C.J. Dale, C.K. Pan, J.L. Flinner, W.K. Chu, and T.G. Finstad, J. Appl. Phys. 58/11, 4459 (1985).

47. H. Suzuki, N. Usami, A. Nomura, T. Shishido, K. Nakajima, and T. Suemasu, J. Cryst. Growth 312/22, 3257 (2010).

Publisher's Note Springer Nature remains neutral with regard to jurisdictional claims in published maps and institutional affiliations. 